\title{
Cranial base strategies for resection of craniopharyngioma in children
}

\author{
James K. LiU, M.D., Chad D. Cole, M.D., M.Sc., John R. W. Kestle, M.D., \\ Douglas L. Brockmeyer, M.D., and Marion L. Walker, M.D. \\ Department of Neurosurgery, University of Utah School of Medicine, Primary Children's Medical \\ Center, Salt Lake City, Utah
}

\begin{abstract}
The optimal treatment of craniopharyngioma in children remains a challenge. The use of complete excision to minimize recurrence continues to be controversial because of the risk of postoperative morbidity and death. Advances in skull base approaches, modern microsurgical techniques, neuroimaging, and hormone replacement therapy, however, have allowed safe gross- or near-total resection in the majority of cases. Total removal of these tumors, if possible, offers the best chance of cure for the patient. Although craniopharyngiomas are not strictly tumors of skull base origin, their intimate relationship with the neurovascular structures of this region often requires a skull base approach to maximize the surgical corridor and facilitate adequate microsurgical resection. In this review, the authors focus on commonly used skull base approaches for the surgical management of craniopharyngioma. They discuss the relative indications, advantages, disadvantages, and complications associated with each approach. Illustrative cases and intraoperative videos are presented.
\end{abstract}

\section{KEY WORDS • craniopharyngioma $・$ skull base surgery $・$ surgical approach}

Craniopharyngiomas are benign tumors that arise from embryonic squamous cells of the craniopharyngeal duct (Rathke pouch). $6,29,38,39,58$ The substantial variability in the location of these tumors is determined by embryological events in the sellar-suprasellar region. ${ }^{5,44}$ Although craniopharyngiomas arise primarily in the sellar and suprasellar regions, they can appear anywhere along the developmental path of the Rathke pouch. ${ }^{34}$ Despite their benign histopathological features, craniopharyngiomas are usually very adherent to the pituitary stalk and may infiltrate the region of the tuber cinereum and hypothalamus, rendering their complete resection hazardous. ${ }^{16}$ These tumors grow by expansion, although small papillary projections and reactive astrogliosis may falsely give the impression of tumor invasion. Craniopharyngiomas may cause compression on parasellar structures such as the optic chiasm and nerves, the pituitary stalk and gland, the floor of the third ventricle, and the cerebral vasculature of the circle of Willis, thereby producing symptoms of visual loss, hypopituitarism, or hydrocephalus. ${ }^{16,57}$

The surgical management of craniopharyngioma remains a challenge to neurosurgeons. The role of complete surgical removal of these tumors is still somewhat controversial. Although some favor radical resection, others have advocated less aggressive strategies, such as stereotactic cyst aspiration, intracystic brachytherapy, and stereotactic radiosurgery. These strategies may be used in a multimodal fashion to provide tumor control in select cases; however,

Abbreviations used in this paper: $\mathrm{CT}=$ computerized tomography; MR = magnetic resonance. this may not eliminate the tumor entirely. Hoffman, et al., ${ }^{17}$ advocated total excision whenever possible and achieved gross-total resection in the majority of cases in their series. Yaşargil, et al., ${ }^{58}$ promoted the strategy of complete tumor removal rather than risking repeated surgical procedures and/or radiation therapy for tumor recurrences. Symon and Sprich $^{51}$ also argued that obtaining a radical resection of all tumor that was accessible and visible microsurgically was best achieved at the first operation. In the experience reported by Fahlbusch, et al.,${ }^{14}$ total resection was attempted; however, subtotal or partial resection was performed if intraoperative findings indicated a significant risk of injuring critical neurovascular structures with radical resection. We agree that complete microsurgical removal, when safe, is the treatment of choice that offers the best chance of cure.

With advances in microsurgical and skull base techniques, safe gross- or near-total excision of these tumors has become possible in the majority of cases, and these procedures are associated with low rates of morbidity and mortality. ${ }^{1,16,17,38,41}$ This treatment method has also been facilitated with adequate hormone replacement therapy. Although craniopharyngiomas are not strictly tumors of skull base origin, they are usually intimately involved with several of its areas, which often necessitates a skull base approach when resecting them. ${ }^{34,52}$ In this review, we present the different cranial base surgical strategies in the resection of craniopharyngiomas, and discuss their respective advantages and disadvantages and relative indications. Other strategies, such as stereotactic cyst aspiration, intracystic brachytherapy, and stereotactic radiosurgery will not be discussed. 


\section{PREOPERATIVE CONSIDERATIONS}

\section{Tumor Classification}

The extent and location of the tumor must be clarified before choosing the appropriate approach. The variability in location of craniopharyngiomas with respect to their surrounding structures has resulted in numerous topographical classifications. ${ }^{7,8,17,26,43,47,48,58}$ Most of these classification systems are based on a vertical projection of tumor growth with respect to the sella turcica, the optic chiasm, and the floor of the third ventricle. ${ }^{48}$ Hoffman, et al., ${ }^{17}$ classified craniopharyngiomas as intrasellar, prechiasmatic, and retrochiasmatic. Samii and $\mathrm{Samii}^{44}$ divided these tumors into five grades depending on vertical extension: Grade I (tumor is located purely in the intrasellar or infradiaphragmatic region); Grade II (tumor is localized in the cistern with or without an intrasellar component); Grade III (tumor extends into the lower half of the third ventricle); Grade IV (tumor expands to the upper half of the third ventricle); or Grade V (tumor dome reaches the septum pellucidum or extends into the lateral ventricles). These classification systems have facilitated selection of various surgical approaches based on tumor location. ${ }^{7}$ Nevertheless, they are just approximations, and as a practical matter, it may not be easy to classify an individual tumor absolutely according to these groups, because tumor growth may arise from several points along the hypophysial axis. ${ }^{27}$

\section{Neuroimaging Studies}

The neuroimaging modality of choice is MR imaging, because of its precise demonstration of the extent and location of the tumor as well as the lesion's relationship to important surrounding neurovascular structures. ${ }^{7}$ It is important to determine the extent of intrasellar or suprasellar involvement and whether the tumor is prechiasmatic or retrochiasmatic, and to identify the presence of intraventricular involvement and posterior fossa extension. The use of MR imaging is also helpful in determining solid and cystic components of the tumor. Recognizing the presence of hydrocephalus is important for preoperative planning, because some patients may require external ventricular drainage before surgery. Although MR imaging offers detailed anatomical information, the presence of adhesions cannot be ascertained by viewing these images and may only be detected at the time of surgical exploration. ${ }^{58}$ The use of MR angiography is helpful in demonstrating the course and relationship of the cerebral vasculature. The vessels of the circle of Willis may be displaced or sometimes engulfed by larger tumors. The use of CT scanning is beneficial in demonstrating the extent of calcifications in the tumor and the osseous changes in the skull base.

\section{Endocrinological Evaluation}

The endocrine status of the patient must be evaluated before surgery is performed for lesions near the hypothalamic-hypophysial axis. Preoperative endocrine evaluation should include measurement of growth hormone, insulinlike growth factor-I, serum prolactin, morning cortisol, thyroid-stimulating hormone, thyroxine, triiodothyronine, follicle-stimulating hormone, luteinizing hormone, testosterone in males, and estradiol in females. Patients with an inadequate pituitary reserve are at risk for intraoperative or postoperative hypopituitarism, which can be dangerous in the perioperative period.

The two most important hormone axes are those related to cortisol and thyroid production. A thyroid function profile and a baseline cortisol level should be obtained preoperatively in anticipation of intraoperative manipulation of the hypothalamic-hypophysial axis. The risk of hypocortisolemia is controlled by the concomitant use of exogenous glucocorticoid agents. Preoperative recognition of hypothyroidism is also important because it can manifest acutely during the early postoperative period. Ideally, patients should be given oral hormone replacements approximately 1 week before surgery to establish a euthyroid state. In urgent or emergency cases, intravenous hormone replacement may be undertaken. In children, short stature or retarded linear growth are sometimes present because of growth hormone deficiency. In adolescents, delayed or arrested puberty may be observed. Mild hyperprolactinemia is usually a result of hypothalamic-hypophysial disconnection, or the "stalk effect." Diabetes insipidus and the syndrome of inappropriate antidiuretic hormone secretion are also seen in the pediatric population. ${ }^{52}$

\section{Neuro-Ophthalmological Evaluation}

Because visual disturbances frequently occur in patients harboring craniopharyngiomas, a formal neuro-ophthalmological examination is warranted preoperatively. This serves as a baseline for comparison of visual status after surgery. The examination should include both visual acuity and visual field testing. A funduscopic examination should also be performed to look for papilledema and optic atrophy. Clinical manifestations of visual compromise may include decreased visual acuity, diplopia, blurred vision, bitemporal hemianopia, central scotomas, see-saw nystagmus, and blindness..$^{51,52}$

\section{Choosing the Appropriate Surgical Approach}

Many approaches have been promoted for craniopharyngiomas. Choosing the appropriate skull base approach depends primarily on the location of the lesion. The approach must provide exposure that creates the shortest distance to the lesion, adequate visualization of the lesion, control of critical neurovascular structures, and minimal brain retraction. A surgical strategy can be established using the MR imaging and CT findings. Additionally, the concepts of keyhole microneurosurgery should be applied by removing or mobilizing additional bone at the outer surface of the skull, such as the supraorbital bar or the zygoma, so that a wider exposure at the surface of the skull will improve viewing trajectories and instrument maneuverability. A "minimally invasive approach" should translate as an approach that provides maximal exposure and minimal damage to the neural structures rather than as simply a "small cosmetic incision."

\section{SURGICAL APPROACHES}

Although various skull base approaches have been described for the excision of craniopharyngiomas, these can be simplified into the following: anterior midline (subfrontal, transsphenoidal); anterolateral (pterional, orbitozygomatic); and intraventricular (transcallosal-transventricu- 
lar, transcortical-transventricular, translamina terminalis) approaches. Variations of each approach can be tailored to the patient by modifying the degree of bone removal. Furthermore, approaches can also be used in combination for extensive lesions that cannot be adequately accessed via a single approach.

\section{Subfrontal-Translamina Terminalis Approach}

Advantages and Disadvantages. The subfrontal approach, also referred to as the bifrontal transbasal approach, is a versatile procedure for removing craniopharyngiomas that are strictly midline with extension along the anterior skull base and suprasellar cistern (Fig. 1). ${ }^{10}$ This approach has the advantage of a straight frontal trajectory with good control of both optic nerves and internal carotid arteries. It also has the advantage of constituting a direct approach through the lamina terminalis for access to the anterior third ventricle if there is intraventricular extension of the tumor. ${ }^{3,49}$ This approach also allows the prechiasmatic portion of the craniopharyngioma to be dissected easily between the optic nerves. Furthermore, any retrochiasmatic portion of the tumor can be excised through the opticocarotid space and the lamina terminalis. ${ }^{7}$ The disadvantages of this approach include potential violation of the frontal sinus and damage to the olfactory tracts. This procedure is usually not recommended in patients with a prefixed chiasm.

Description of Procedure. The patient is positioned supine with the head elevated $30^{\circ}$ to facilitate venous drainage (Fig. 1). The head is extended to help with frontal lobe relaxation. Lumbar drainage may be used for additional brain relaxation. After a bicoronal incision is made, the scalp flap is reflected anteriorly, followed by a bifrontal craniotomy. The inferior limit of the bone flap should be at the level of the orbital roof to maximize surgical exposure and minimize brain retraction. Harvesting a vascularized pericranial flap from the scalp for subsequent reconstruction of the cranial base at the time of closure is an important step in this procedure. Access to the anterior skull base is less difficult in children than in adults because its floor is shallow and relatively foreshortened and the frontal sinuses are not fully developed in children. Therefore, we rarely remove the supraorbital bar (extended subfrontal approach) in these cases. If, however, the frontal sinuses are violated, exenteration and cranialization of the frontal sinuses followed by plugging of the nasofrontal ducts with muscle and bone chips is performed. The dura mater is opened transversely a few millimeters behind the supraorbital bar. The superior sagittal sinus is ligated anteriorly with a suture and divided along the plane of the dural incision at the insertion of the falx cerebri.

After adequate brain relaxation is obtained, the frontal lobes are gently held by self-retaining retractors. Care should be taken to avoid excessive brain retraction to prevent postoperative edema or ischemia. ${ }^{22}$ The olfactory tracts are freed from their arachnoid attachments. The right olfactory tract is divided behind the olfactory bulb, providing exposure of the optic nerves. The chiasmatic and interhemispheric cisterns are opened carefully to expose the optic nerves and chiasm, the lamina terminalis, the anterior communicating artery complex, and the internal carotid arteries. The optic nerves and chiasm must be carefully dissected from the capsule of the craniopharyngioma. Tumors

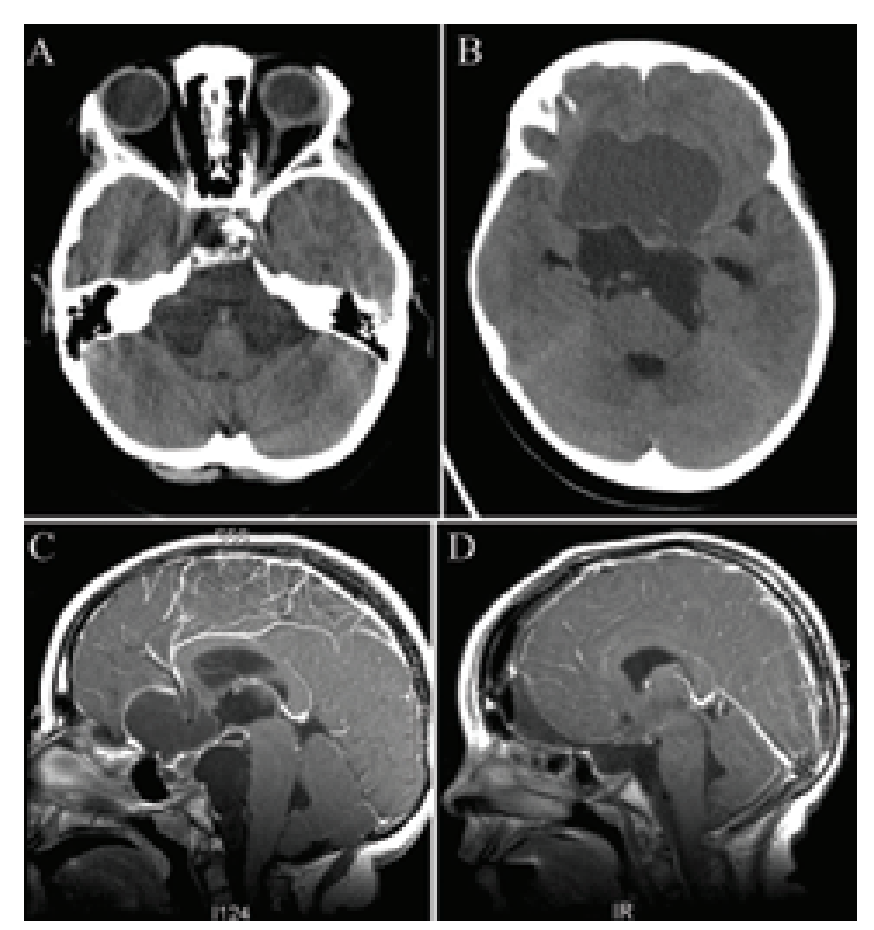

Fig. 1. Preoperative $\mathrm{CT}(\mathrm{A}$ and $\mathrm{B})$ and $\mathrm{MR}$ images $\left(\mathrm{C}: \mathrm{T}_{1}-\right.$ weighted post-Gd sagittal MR image) of an extensive craniopharyngioma in a 9-year-old boy who presented with headaches, growth retardation, bitemporal hemianopia, and bilateral papilledema. The tumor occupied the anterior skull base, the intrasellar and suprasellar region, and the retrochiasmatic region and extended into the retroclival prepontine space. A subfrontal approach was used to achieve a gross-total resection (D: postoperative $\mathrm{T}_{1}$-weighted post-Gd sagittal MR image).

in the prechiasmatic, opticocarotid, and carotid-oculomotor cisterns are readily accessible via the subfrontal approach. Cystic lesions are initially decompressed by opening the capsule. To prevent aseptic meningitis, care should be taken to prevent dissemination of intracystic contents to the subarachnoid space. Additionally, occlusion of the cerebral aqueduct by keratin and cholesterol found within the craniopharyngioma may result in hydrocephalus., ${ }^{719}$ The capsule is then carefully dissected away from adherent neurovascular structures by using the microsurgical technique. Incomplete removal of the capsule may result in tumor recurrence.

Tools. Removal of intrasellar tumor is possible but direct visualization into the sellar floor is limited. We use angled endoscopes or dental mirrors to inspect for residual tumor in the sella turcica. These tools are also useful for inspecting the undersurface of the optic nerves and chiasm. It may be possible to identify and preserve the pituitary stalk in patients with small suprasellar tumors, but this becomes more difficult with large lesions and may not be feasible because of the potential for leaving residual tumor behind. Once the prechiasmatic and suprasellar portions of the tumor have been removed, direct access into the retrosellar and retroclival spaces can be attained, if needed. Craniopharyngiomas that extend into the posterior fossa can be removed (Fig. 1, Video 1).

Click here to view the video clip: Removal of an extensive 
craniopharyngioma by using the subfrontal approach in the patient whose tumor is depicted in Fig. 1.

For retrochiasmatic craniopharyngiomas that reside within the third ventricle, a translamina terminalis approach is used. The lamina terminalis is situated between the optic tracts and extends from the anterior commissure to the posterior limit of the optic chiasm. This forms the inferior two thirds of the anterior wall of the third ventricle. To access the tumor within the third ventricle, an incision in the lamina terminalis is made in the anteriormost part of the ventricle, immediately posterior to the chiasm. This incision is extended from one optic tract to the other while staying below the anterior communicating artery. After the lamina terminalis is opened, the tumor can be identified and carefully dissected away from structures of the anterior hypothalamus. Care must be taken to limit excessive retraction and damage to perforating vessels originating from the anterior cerebral artery. ${ }^{39}$ This approach is useful if significant intraventricular extension is present.

\section{Transsphenoidal and Extended Transsphenoidal} Approaches

Advantages and Disadvantages. Transsphenoidal resection is favored for craniopharyngiomas that occupy both sellar and suprasellar regions, especially if the sella turcica is enlarged. 4,8,12,14,18,21,23,28,30,32,38 This approach is most appropriate for intrasellar and subdiaphragmatic craniopharyngiomas. An intrasellar location with enlargement of the sella turcica and a rounded suprasellar extension indicates a subdiaphragmatic craniopharyngioma. ${ }^{14}$ Suprasellar extensions of tumors may be readily removed with the transsphenoidal approach, given that they are primarily cystic and not solid. Both solid and cystic tumors in the intrasellar portion can be removed with the transsphenoidal approach.

If the tumor is purely suprasellar with a normal-sized sella turcica, extended variations such as the transsellartransdiaphragmatic approach or the transsphenoidaltranstuberculum approach can sometimes be used. ${ }^{12,21,23,28 \text {, }}$ ${ }^{32,36}$ By changing the trajectory of the nasal speculum and removing additional bone from the skull base, an extended transsphenoidal approach can allow additional exposure despite the presence of a normal sella turcica. A transsellar-transdiaphragmatic method of approaching the suprasellar cisterns may be used in the surgical removal of craniopharyngiomas. ${ }^{14,21,32}$ In the transsphenoidal-transtuberculum approach, bone is removed from the sellar floor, tuberculum sellae, and posterior part of the planum sphenoidale for access to the contents of the basal cisterns. ${ }^{14,21}$, 28,38 This approach provides direct access to supradiaphragmatic craniopharyngiomas adjacent or anterior to the pituitary stalk, without resecting the pituitary gland. ${ }^{7,38}$ In these cases, the additional bone exposure provides improved visualization of the suprasellar portions of the tumor and reduces the amount of blind curettage in this region. Cystic tumors are particularly amenable to drainage and removal by this approach. Excellent results in craniopharyngiomas resected via the transsphenoidal route or an extended variation have been reported by Laws and colleagues, ${ }^{21,32}$ Maira, et al., ${ }^{38}$ and Couldwell, et al. ${ }^{12}$ The transsphenoidal approach may not be suitable for cases in which there is significant lateral extension. In these cases, a frontotemporal or a combined approach may be necessary.
The transsphenoidal approach is more difficult in young children who do not have a pneumatized sphenoid sinus. In these cases, access to the sella turcica requires additional drilling of the sphenoid bone with the aid of stereotactic CT guidance. ${ }^{20,42}$ In general, we prefer not to use the transsphenoidal approach as the primary one if the sphenoid sinus is not favorably pneumatized.

The transsphenoidal approach offers a midline exposure, allowing dissection in the space around the optic nerves, avoiding brain retraction and some of the other disadvantages of transcranial surgery. ${ }^{7,28}$ Only the propensity for CSF leakage was slightly increased for craniopharyngioma removal compared with other standard transsphenoidal procedures. ${ }^{38}$ Additionally, the risk of visual injury was found to be reduced when compared with craniotomies performed to treat similar lesions. ${ }^{18,32,58}$ Craniopharyngiomas involving the sella turcica are particularly amenable to the transsphenoidal approach because tumors in this location are most often cystic or friable. ${ }^{18,30,38}$ Furthermore, craniopharyngiomas located within this region do not infiltrate surrounding structures, making tumor debulking and capsule dissection from the optic chiasm, hypothalamus, and pituitary stalk practicable. ${ }^{38}$ This regional characteristic is in stark contrast to those infundibular craniopharyngiomas that are more often calcified and those intraventricular craniopharyngiomas that are almost always solid. ${ }^{39,40}$

Description of Procedure. The patient is placed supine on the operating table with the head elevated approximately $15^{\circ}$ on a horseshoe headrest. In the standard transsphenoidal-transsellar approach, the patient's face is placed parallel to the ceiling. For the extended transsphenoidal approach, the head is further extended to allow visualization of the anterior skull base and suprasellar region. The sella turcica can be exposed with either a sublabial or an endonasal ap-

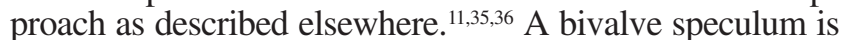
placed, the sphenoid sinus is opened using Kerrison rongeurs, and all sphenoid mucosa is exenterated. The sellar floor is then removed with Kerrison rongeurs to expose the sellar dura mater. For the extended transsphenoidal approach, the bone of the tuberculum sellae is removed by first removing a small amount of bone over the anterior sellar wall to expose the anterior circular sinus and then extending this bone removal rostrally by using microrongeurs. The planum sphenoidale can be removed more anteriorly to provide additional exposure of the suprasellar region. One must remain cognizant of the position of the circular sinus, which demarcates the anterior extension of the sella turcica, the anterior communicating artery complex superiorly, the ethmoid sinuses anteriorly, and the optic nerves superolaterally. Once the bone of the tuberculum sellae has been removed, the dura mater anterior and inferior to the circular sinus is opened in the midline. The sinus is then coagulated and transected to gain a direct view of the suprasellar cistern while preserving the pituitary gland in its position. This approach enables an unencumbered view of the suprasellar cistern above the pituitary gland.

After tumor removal, the dural defect must be repaired carefully with autologous fascia lata and fat. The fat is placed in the bony opening of the skull base, followed by fascia lata to cover the dural defect. A piece of Marlex mesh can be used to repair the skull base defect. The repair is buttressed in place and supported by packing the sphenoid sinus region with fat. Closure of the mucosal incisions is per- 
formed in the usual fashion as described previously. ${ }^{35,36}$ For large skull base defects, lumbar drainage is used for several days postoperatively.

Even though the transsphenoidal route is useful for craniopharyngiomas occurring predominantly within the sella turcica with or without marked suprasellar expansion, its use in association with a transcranial approach has some important advantages, especially if the tumor spreads far beyond the sella. ${ }^{27}$ In this case, the transsphenoidal route may be used before the intracranial approach, ${ }^{24}$ in combination with it, ${ }^{25}$ or afterward. Some surgeons recommend an interval of 3 months between operations. ${ }^{24}$ If a transcranial route is considered necessary in conjunction with a transsphenoidal approach, the majority of tumors can be removed with a subfrontal or pterional approach.

\section{Pterional Approach}

Advantages and Disadvantages. The pterional (frontotemporal) approach is the workhorse for the surgical treatment of craniopharyngiomas involving primarily the suprasellar cistern..$^{58}$ This approach provides the shortest distance to the suprasellar region for a transcranial approach. This exposure is suitable for removing craniopharyngiomas involving the intrasellar, suprasellar, prechiasmatic, and retrochiasmatic regions (Fig. 2). This is also the preferred method in patients with a prefixed chiasm, because the tumor can be resected beneath the chiasm.

A disadvantage of the pterional approach is the limited view of the contralateral opticocarotid triangle and the contralateral retrocarotid space. ${ }^{7}$ Additionally, when the lamina terminalis is accessed through a pterional craniotomy, the oblique trajectory of this route makes it difficult to visualize the posterior part of the third ventricle, especially the ipsilateral lateral wall of the hypothalamus. ${ }^{5}$ This places the columns of fornix, supraoptic nuclei, organ vasculosum, and tuber cinereum at risk for retraction injury or perforating vessel damage. ${ }^{13,39}$ If the surgeon anticipates that the craniopharyngioma will be significantly extended posteriorly within the third ventricle, a transcallosal- or transcortical-transventricular approach allows better access. ${ }^{5}$

Description of Procedure. The patient is positioned supine with the head rotated 30 to $45^{\circ}$ to the left and the neck extended 15 to $20^{\circ}$ so that the malar eminence is at the highest point. This maneuver allows the frontal lobe to fall away from the skull base. Additional brain relaxation can be achieved with a lumbar or external ventricular drain. A frontotemporal incision is made beginning in front of the ear at the level of the zygoma and extending toward the midline behind the hairline. The temporalis muscle can be mobilized as a myocutaneous flap or as separate layers by using an interfascial or subfascial muscle dissection. ${ }^{9,59} \mathrm{It}$ is important not to violate the frontalis branch of the facial nerve. In younger children, the temporalis muscle is often not bulky, so a myocutaneous flap is usually sufficient. A frontotemporal bone flap is elevated and the lesser wing of the sphenoid is drilled down to optimize the most basal trajectory to the skull base. The bone flap can be extended more medially to provide a more subfrontal trajectory to tumors extending between the optic nerves. The anterior clinoid process is removed if access to the anterior cavernous sinus or paraclinoid region is needed. The dura mater is then opened with a C-shaped incision centered on the

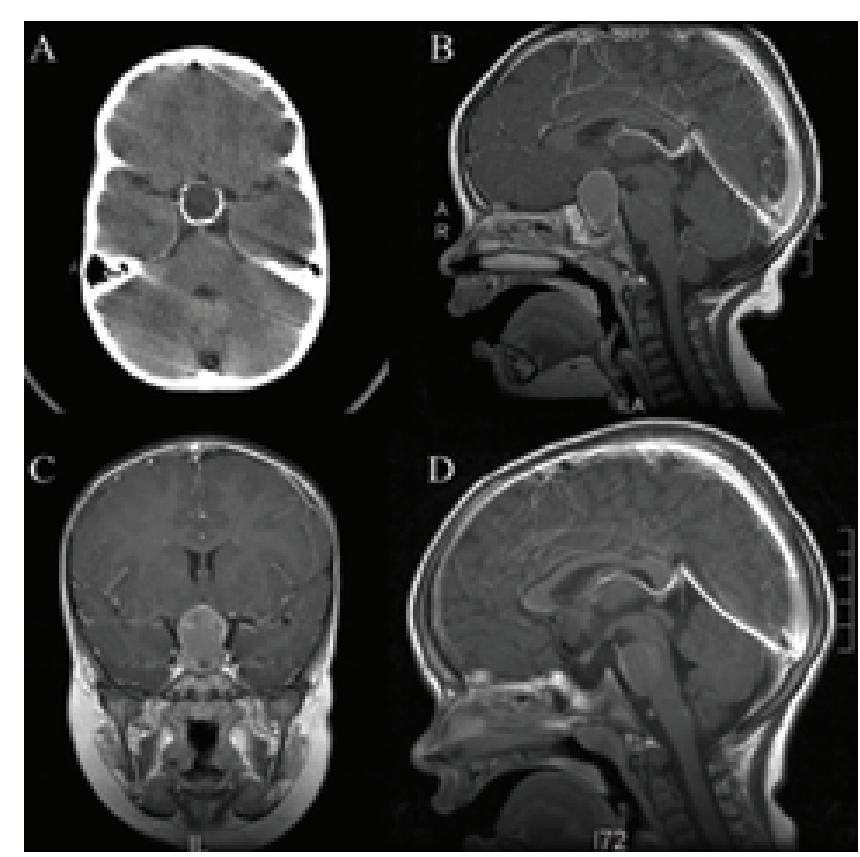

Fig. 2. Preoperative CT (A) and MR images (B and C: sagittal and coronal $\mathrm{T}_{1}$-weighted post-Gd MR images) of an intrasellar and suprasellar craniopharyngioma in a 2-year-old boy that was resected using a pterional approach. The postoperative $\mathrm{T}_{1}$-weighted postGd sagittal MR image (D) demonstrates a gross-total resection.

sphenoid wing. Wide splitting of the sylvian fissure is performed and CSF is released from the cisterns, which exposes the parachiasmal spaces.

Depending on the location and extension of the tumor, its extirpation may be performed through the opticocarotid, carotid-oculomotor, and prechiasmatic spaces. Attention is paid to avoid violating the perforating arteries situated in the opticocarotid triangle. Exposure of the anteroinferior third ventricle can be performed by opening the lamina terminalis, as described earlier, for access to craniopharyngiomas of the third ventricle. ${ }^{39}$ The craniopharyngioma can be carefully dissected away from the anterolateral neural structures and walls of the third ventricle, with careful attention directed to preservation of the visual pathways and the hypothalamic structures. Endoscopy and angled pituitary curettes may be used to remove the more posteriorly situated tumor?

\section{Orbitozygomatic Approach}

Advantages and Disadvantages. The orbitozygomatic approach is essentially a pterional craniotomy with removal of the supraorbital rim, zygomatic arch, or both. ${ }^{33,60}$ The orbitozygomatic approach provides a wider exposure at the surface of the cranium to allow more freedom to maneuver surgical instruments, and it improves the angles of exposure of the posterior clinoid, basilar apex, and suprasellar region. ${ }^{15}$ This approach is useful for resecting craniopharyngiomas with significant suprasellar extension because it offers an improved inferior-to-superior ("looking-up") view to the hypothalamic and suprasellar region (Fig. 3, Video 2 ). Removal of the zygomatic arch allows more inferior mobilization of the temporalis muscle and reduces the muscle bulk that may otherwise obstruct visualization. 
Click here to view the video clip: Removal of a suprasellar crantopharyngionna by using the Inodified orbitozygomatic approach in the patient whose tumor is depicted in Fig. 3.

The orbitozygomatic craniotomy is also very effective in approaching those craniopharyngiomas that have significant superior extension into the third ventricle. By removing the orbital rim and lateral sphenoid region, the obstructing bone that typically limits adequate exposure of the superior third ventricle through the lamina terminalis or underneath the $A_{1}$ portion of the anterior cerebral artery is circumvented. In essence, the angle of exposure, based on the fulcrum of the inferior frontal lobe, is significantly improved. ${ }^{45}$

Description of Procedure. The patient positioning and skin incision are similar to that of the pterional approach described earlier. A subfascial dissection of the temporalis muscle is performed to expose the orbitozygomatic complex. ${ }^{9,60}$ This complex can be removed with the frontotemporal bone flap as one piece or it can be removed after fashioning the bone flap as two pieces. Alternatively, a modified orbitozygomatic approach (removal of the supraorbital rim or zygomatic arch only) can be performed, depending on the location of the pathological entity (Fig. 3, Video 2). ${ }^{33}$ Care should be taken not to violate the temporomandibular joint during the zygomatic osteotomy. The remainder of the surgical approach is similar to that of the pterional approach described earlier.

\section{Transcallosal-Transventricular Approach}

Advantages and Disadvantages. The anterior transcallosal approach provides direct access to the lateral ventricle and the foramen of Monro with minimal retraction of the brain. $29,31,37,55$ This approach provides good exposure of extensive craniopharyngiomas that occupy the anterior third ventricle and project into the lateral ventricle through the foramen of Monro. This foramen serves as a corridor into the anterior and middle portions of the third ventricle, particularly when the foramen is dilated by the tumor. ${ }^{50}$ When the exposure through the foramen of Monro is limited, other maneuvers, such as subchoroidal exposure, ${ }^{54}$ transchoroidal exposure, ${ }^{56}$ or interforniceal exposure, ${ }^{2}$ are required to gain access to the third ventricle. This approach can be used in combination with a subfrontal or frontotemporal one (pterional or orbitozygomatic) for large suprasellar and intraventricular craniopharyngiomas (Fig. 4), ${ }^{4,37}$ The transcallosal approach permits removal of the intraventricular portion of the tumor as the first stage. Subsequently, if the basal portion of the tumor remains inaccessible, the subfrontal or frontotemporal approach may be used to extirpate the extraventricular part of the craniopharyngioma. ${ }^{27}$

The transcallosal approach offers the advantages of a shorter distance to the third ventricle than with the transcortical approach, greater flexibility to explore the entire anterior-posterior extent of the third ventricle without disruption of hemispheral tissue, no cortical incision, and an

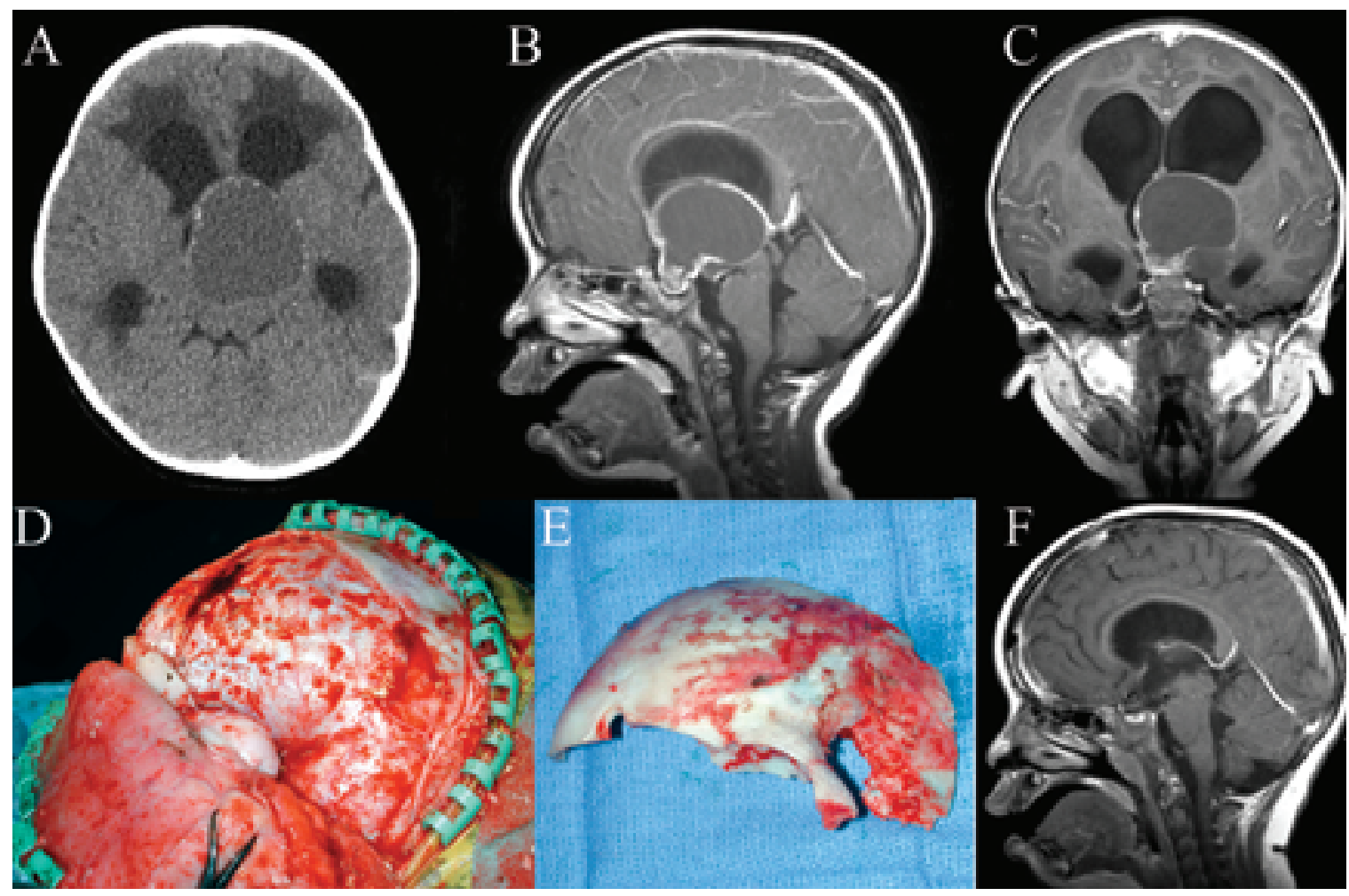

Fig. 3. Preoperative CT (A) and MR images (B and C: sagittal and coronal $\mathrm{T}_{1}$-weighted post-Gd MR images) of a large craniopharyngioma in a 4-year-old girl that was resected using a modified orbitozygomatic approach. D and E: Intraoperative photographs demonstrating the one-piece modified orbitozygomatic approach with removal of the supraorbital rim. The bone flap was extended frontally to allow a combined subfrontal and frontotemporal trajectory. F: Postoperative $\mathrm{T}_{1}$-weighted post-Gd sagittal MR image demonstrating a gross-total resection. 
unobstructed view to the depths of the anterior third ventricle. ${ }^{46}$ If the lateral ventricles are small, the transcallosal approach is preferred over the transcortical one, because it is not dependent on enlarged lateral ventricles for access. This approach offers limited control of the suprasellar region. Other major risks include retraction injury to the cerebral hemispheres; venous infarction from injury to the veins draining into the superior sagittal sinus; injury to the pericallosal arteries; injury to the fornix and anterior commissure; and injury to the septal, thalamostriate, and internal cerebral veins. ${ }^{7}$

Description of Procedure. The patient is placed supine with the head tilted up $30^{\circ}$. The use of MR stereotactic guidance is helpful in planning the craniotomy and callosal incision. An incision is made at the level of the coronal suture crossing the midline. ${ }^{7,27}$ This incision can be extended inferiorly toward the zygoma if a combined approach is planned. A small bilateral bone flap allows good visualization of the interhemispheral space. ${ }^{7}$ The dura mater is opened with a $\mathrm{C}$-shaped incision and reflected medially over the superior sagittal sinus. The right cerebral hemisphere is gently retracted laterally. Venous infarction from injury to the superficial bridging veins is prevented by using intermittent retraction of the cerebral hemispheres and minimizing coagulation of the bridging veins. The corpus callosum is opened for approximately 15 to $20 \mathrm{~mm}$, between the pericallosal arteries. Once the lateral ventricle is entered, the following anatomical landmarks should be identified to calculate the surgical orientation: the choroid plexus, foramen of Monro, septum pellucidum, and thalamostriate vein.

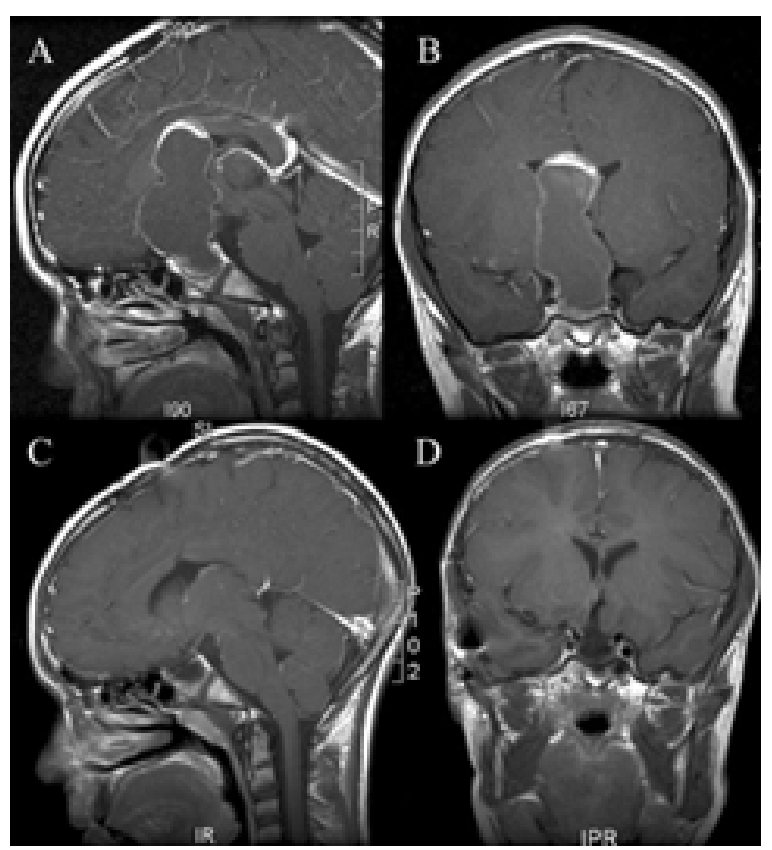

Fig. 4. Preoperative $T_{1}$-weighted post-Gd MR images (A: sagittal view; $\mathrm{B}$ : coronal view) of a giant craniopharyngioma in a 14-year-old boy that extended from the sellar floor to the corpus callosum. The tumor was resected using a combined approach (transcallosal-transventricular approach followed by an orbitozygomatic approach). Postoperative MR images (C: sagittal view; D: coronal view) demonstrate a gross-total resection.
Access into the third ventricle may be achieved through the foramen of Monro; if additional exposure is needed, either the subchoroidal or the interforniceal approach can be used. ${ }^{2,7}$ The subchoroidal approach ${ }^{53,54}$ is performed by opening the choroidal fissure along the tenia choroidea and retracting the choroid plexus and body of the fornix medially to expose the velum interpositum, which is then opened between the thalamus and the ipsilateral internal cerebral vein. The disadvantages of this approach are the risk of damage to the thalamus, the thalamostriate vein, and the choroidal arteries. The interforniceal approach ${ }^{2,53}$ is performed by entering the natural plane between the fornices that opens into the roof of the third ventricle. This interforniceal division must be made exactly in the midline. It is important not to make the incision beyond the region of the interface between the column of the fornix and the anterior commissure. This approach has the risk of creating bilateral forniceal injury and memory loss.

\section{Transcortical-Transventricular Approach}

Advantages and Disadvantages. The transcortical-transventricular approach is an alternative access to third ventricular craniopharyngiomas. This approach is favored if the lateral ventricles are enlarged from hydrocephalus, because it provides the surgical corridor with minimal cortical retraction. The advantages of transcortical-transventricular exposure are that there is less risk of compromising an essential draining vein going to the sagittal sinus and less chance of injuring the pericallosal arteries. ${ }^{46}$ This approach requires violation of the cortex, however, which increases the risk of postoperative seizures. Risk of injury to the fornix, thalamus, and deep venous structures is still a consideration when accessing the third ventricle.

Description of Procedure. The positioning of the patient is the same as in the transcallosal approach. The use of MR stereotactic guidance is helpful in planning the transcortical trajectory to the lateral ventricle. The approach is performed on the nondominant side unless the tumor is located primarily in the contralateral lateral ventricle. Either a coronal or a horseshoe incision is made to expose the frontal region anterior to the coronal suture. A rectangular bone flap is elevated over the region of the planned corticectomy. The dura mater is opened with a $\mathrm{C}$-shaped incision and reflected medially toward the midline. A cortical incision is made paralleling the course of, and in the center of, the middle frontal gyrus for approximately 3 to $4 \mathrm{~cm} .{ }^{46}$ The white matter is then dissected toward the lateral ventricle by using blunt dissection with malleable retractors. The plane of dissection is directed toward the foramen of Monro. Once the lateral ventricle is entered, self-retaining retractors are placed to obtain the exposure. At this juncture, the operation is similar to the transcallosal approach. Additional exposure of the third ventricle can be obtained using either the subchoroidal or the interforniceal approach, as described earlier.

\section{CONCLUSIONS}

Skull base and microsurgical techniques are an important addition to the armamentarium for the surgical management of craniopharyngioma in children. If feasible, grosstotal resection offers the best chance of cure. The recur- 
rence rate is dependent on the extent of resection, which is dependent on the location, consistency (solid or cystic), and size of the tumor and its adhesiveness to critical neurovascular structures. Preoperative neuroimaging is important in determining the location and extent of the tumor and to plan the optimal surgical approach. A combined approach may be required to achieve total resection.

\section{References}

1. Amacher AL: Craniopharyngioma: the controversy regarding radiotherapy. Childs Brain 6:57-64, 1980

2. Apuzzo ML, Chikovani OK, Gott PS, et al: Transcallosal, interfornicial approaches for lesions affecting the third ventricle: surgical considerations and consequences. Neurosurgery 10: 547-554, 1982

3. Asano T: [Interhemispheric, trans-lamina terminalis approach for craniopharyngioma.] No Shinkei Geka 17:799-812, 1989 (Jpn)

4. Baskin DS, Wilson CB: Surgical management of craniopharyngiomas. A review of 74 cases. J Neurosurg 65:22-27, 1986

5. Behari S, Banerji D, Mishra A, et al: Intrinsic third ventricular craniopharyngiomas: report on six cases and a review of the literature. Surg Neurol 60:245-253, 2003

6. Bose B, Huang P, Myers D, et al: Intrinsic third ventricular craniopharyngioma: two case reports with review of the literature. Del Med J 57:389-394, 1985

7. Choux M, Lena G: Craniopharyngioma, in Apuzzo MLJ (ed): Surgery of the Third Ventricle, ed 2. Baltimore: Williams \& Wilkins, 1998, pp 1143-1181

8. Ciric IS, Cozzens JW: Craniopharyngiomas: transsphenoidal method of approach-for the virtuoso only? Clin Neurosurg 27:169-187, 1980

9. Coscarella E, Vishteh AG, Spetzler RF, et al: Subfascial and submuscular methods of temporal muscle dissection and their relationship to the frontal branch of the facial nerve. Technical note. J Neurosurg 92:877-880, 2000

10. Couldwell WT: Surgery of the anterior skull base. Otolaryngol Clin North Am 26:673-693, 1993

11. Couldwell WT: Transsphenoidal and transcranial surgery for pituitary adenomas. J Neurooncol 69:237-256, 2004

12. Couldwell WT, Weiss MH, Rabb C, et al: Variations on the standard transsphenoidal approach to the sellar region, with emphasis on the extended approaches and parasellar approaches: surgical experience in 105 cases. Neurosurgery 55:539-550, 2004

13. de Divitiis O, Flavio Angileri FF, d'Avella D, et al: Microsurgical anatomic features of the lamina terminalis. Neurosurgery 50:563-570, 2002

14. Fahlbusch R, Honegger J, Paulus W, et al: Surgical treatment of craniopharyngiomas: experience with 168 patients. J Neurosurg 90:237-250, 1999

15. Gonzalez LF, Crawford NR, Horgan MA, et al: Working area and angle of attack in three cranial base approaches: pterional, orbitozygomatic, and maxillary extension of the orbitozygomatic approach. Neurosurgery 50:550-557, 2002

16. Habrand JL, De Crevoisier R: Radiation therapy in the management of childhood brain tumors. Childs Nerv Syst 17:121-133, 2001

17. Hoffman HJ, De Silva M, Humphreys RP, et al: Aggressive surgical management of craniopharyngiomas in children. J Neurosurg 76:47-52, 1992

18. Honegger J, Buchfelder M, Fahlbusch R, et al: Transsphenoidal microsurgery for craniopharyngioma. Surg Neurol 37:189-196, 1992

19. Horoupian DS, Wisniewski HM, Gamble R, et al: Aqueduct gliosis caused by keratin and cholesterol in a case of craniopharyngioma. Can J Neurol Sci 1:185-188, 1974

20. Im SH, Wang KC, Kim SK, et al: Transsphenoidal microsurgery for pediatric craniopharyngioma: special considerations regard- ing indications and method. Pediatr Neurosurg 39:97-103, 2003

21. Kaptain GJ, Vincent DA, Sheehan JP, et al: Transsphenoidal approaches for the extracapsular resection of midline suprasellar and anterior cranial base lesions. Neurosurgery 49:94-101, 2001

22. Kasama A, Kanno T: A pitfall in the interhemispheric translamina terminalis approach for the removal of a craniopharyngioma. Significance of preserving draining veins. Part II. Experimental study. Surg Neurol 32:116-120, 1989

23. Kato T, Sawamura Y, Abe H, et al: Transsphenoidal-transtuberculum sellae approach for supradiaphragmatic tumors: technical note. Acta Neurochir 140:715-719, 1998

24. Kobayashi T, Nakane T, Kageyama N: Combined trans-sphenoidal and intracranial surgery for craniopharyngioma. Prog Exp Tumor Res 30:341-349, 1987

25. Kobayashi T, Tanaka T, Kida Y: Stereotactic gamma radiosurgery of craniopharyngiomas. Pediatr Neurosurg 21 (Suppl 1):69-74, 1994

26. Konovalov AN: Microsurgery of tumours of diencephalic region. Neurosurg Rev 6:37-41, 1983

27. Konovalov AN: Technique and strategies of direct surgical management of craniopharyngiomas, in Apuzzo MLJ (ed): Surgery of the Third Ventricle, ed 2. Baltimore: Williams \& Wilkins, 1998, pp 1133-1142

28. Kouri JG, Chen MY, Watson JC, et al: Resection of suprasellar tumors by using a modified transsphenoidal approach. Report of four cases. J Neurosurg 92:1028-1035, 2000

29. Kunishio K, Yamamoto Y, Sunami N, et al: Craniopharyngioma in the third ventricle: necropsy findings and histogenesis. J Neurol Neurosurg Psychiatry 50:1053-1056, 1987

30. Landolt AM, Zachmann M: Results of transsphenoidal extirpation of craniopharyngiomas and Rathke's cysts. Neurosurgery 28:410-415, 1991

31. Lanzieri CF, Sacher M, Som PM: CT changes in the septum pellucidum associated with intraventricular craniopharyngiomas. J Comput Assist Tomogr 9:507-510, 1985

32. Laws ER Jr: Transsphenoidal microsurgery in the management of craniopharyngioma. J Neurosurg 52:661-666, 1980

33. Lemole GM Jr, Henn JS, Zabramski JM, et al: Modifications to the orbitozygomatic approach. Technical note. J Neurosurg 99:924-930, 2003

34. Levy ML, Khoo LT, Day JD, et al: Optimization of the operative corridor for the resection of craniopharyngiomas in children: the combined frontoorbitozygomatic temporopolar approach. Technical note. Neurosurg Focus 3(6):E5, 1997

35. Liu JK, Orlandi RR, Apfelbaum RI, et al: Novel closure technique for the endonasal transsphenoidal approach. Technical note. J Neurosurg 100:161-164, 2004

36. Liu JK, Weiss MH, Couldwell WT: Surgical approaches to pituitary tumors. Neurosurg Clin N Am 14:93-107, 2003

37. Long DM, Chou SN: Transcallosal removal of cranio-pharyngiomas within the third ventricle. J Neurosurg 39:563-567, 1973

38. Maira G, Anile C, Albanese A, et al: The role of transsphenoidal surgery in the treatment of craniopharyngiomas. J Neurosurg 100:445-451, 2004

39. Maira G, Anile C, Colosimo C, et al: Craniopharyngiomas of the third ventricle: trans-lamina terminalis approach. Neurosurgery 47:857-865, 2000

40. Maira G, Anile C, Rossi GF, et al: Surgical treatment of craniopharyngiomas: an evaluation of the transsphenoidal and pterional approaches. Neurosurgery 36:715-724, 1995

41. Norris JS, Pavaresh M, Afshar F: Primary transsphenoidal microsurgery in the treatment of craniopharyngiomas. Br J Neurosurg 12:305-312, 1998

42. Orvidas LJ, Kasperbauer JL, Meyer FB, et al: Pediatric transseptal transsphenoidal pituitary surgery. Am J Rhinol 14: 265-271, 2000 


\section{Resection of craniopharyngiomas}

43. Rougerie J: What can be expected from the surgical treatment of craniopharyngiomas in children. Report of 92 cases. Childs Brain 5:433-449, 1979

44. Samii M, Samii A: Surgical management of craniopharyngiomas, in Schmidek HH (ed): Schmidek and Sweet Operative Neurological Techniques: Indications, Methods, and Results, ed. 4. Philadelphia: WB Saunders, 2000, pp 489-502

45. Schwartz MS, Anderson GJ, Horgan MA, et al: Quantification of increased exposure resulting from orbital rim and orbitozygomatic osteotomy via the frontotemporal transsylvian approach. J Neurosurg 91:1020-1026, 1999

46. Shucart W: Anterior transcallosal and transcortical approaches, in Apuzzo MLJ (ed): Surgery of the Third Ventricle. Baltimore: Williams \& Wilkins, 1987, pp 303-325

47. Steno J: Microsurgical topography of craniopharyngiomas. Acta Neurochir Suppl 35:94-100, 1985

48. Steno J, Malacek M, Bizik I: Tumor-third ventricular relationships in supradiaphragmatic craniopharyngiomas: correlation of morphological, magnetic resonance imaging, and operative findings. Neurosurgery 54:1051-1060, 2004

49. Suzuki J, Katakura R, Mori T: Interhemispheric approach through the lamina terminalis to tumors of the anterior part of the third ventricle. Surg Neurol 22:157-163, 1984

50. Symon L, Pell M, Yaşargil MG, et al: Surgical techniques in the management of colloid cysts of the third ventricle. Adv Tech Stand Neurosurg 17:121-157, 1990

51. Symon L, Sprich W: Radical excision of craniopharyngioma. Results in 20 patients. J Neurosurg 62:174-181, 1985

52. Tsai EC, Santoreneos S, Rutka JT: Tumors of the skull base in children: review of tumor types and management strategies. Neurosurg Focus 12(5):E1, 2002
53. Türe U, Yaşargil MG, Al-Mefty O: The transcallosal-transforaminal approach to the third ventricle with regard to the venous variations in this region. J Neurosurg 87:706-715, 1997

54. Viale GL, Pau A, Sehrbundt E, et al: The subchoroidal approach to the third ventricle: surgical anatomy according to Galen. Neurosurgery 49:986-991, 2001

55. Villani RM, Tomei G, Bello L, et al: Long-term results of treatment for craniopharyngioma in children. Childs Nerv Syst 13: 397-405, 1997

56. Wen HT, Rhoton AL Jr, de Oliveira E: Transchoroidal approach to the third ventricle: an anatomic study of the choroidal fissure and its clinical application. Neurosurgery 42: 1205-1219, 1998

57. Wisoff JH: Surgical management of recurrent craniopharyngiomas. Pediatr Neurosurg 21 (Suppl 1):108-113, 1994

58. Yaşargil MG, Curcic M, Kis M, et al: Total removal of craniopharyngiomas. Approaches and long-term results in 144 patients. J Neurosurg 73:3-11, 1990

59. Yaşargil MG, Reichman MV, Kubik S: Preservation of the frontotemporal branch of the facial nerve using the interfascial temporalis flap for pterional craniotomy. Technical article. J Neurosurg 67:463-466, 1987

60. Zabramski JM, Kırış T, Sankhla SK, et al: Orbitozygomatic craniotomy. Technical note. J Neurosurg 89:336-341, 1998

Manuscript received May 10, 2005.

Accepted in final form May 23, 2005.

Address reprint requests to: Marion L. Walker, M.D., Department of Neurosurgery, University of Utah, 30 North 1900 East, Suite 3B409, Salt Lake City, Utah 84132. email: marion.walker@hsc.utah.edu. 